\title{
Variance of interburst intervals in burst suppression *
}

\author{
Ahmad Beydoun, Catherine E. Yen and Ivo Drury \\ EEG Laboratory, Department of Neurology, University of Michigan, Ann Arbor, MI (U.S.A.)
}

(Accepted for publication: 22 July 1991)

\begin{abstract}
Summary Each EEG performed over a 3 year period at the University of Michigan with a diagnosis of generalized burst-suppression (BS) was reviewed. Ten EEGs from 10 patients with hypoxic-ischemic encephalopathy (HIE-BS) and 21 records from 8 patients with pentobarbital induced burst-suppression for treatment of status epilepticus (SE-BS) were reviewed. For each EEG, the mean duration of 40 interburst intervals (IBIs) as well as their coefficient of variability were calculated. We found that in the SE-BS group the coefficient of variability of IBI duration was highly correlated with the logarithm of mean IBI duration while in the HIE-BS group, there was no significant correlation between these 2 variables. This suggests that the underlying mechanism causing BS is different in the 2 groups and might be related to a uniform and progressive affection of similar brain structures in the SE-BS group and a more patchy and variable pathology in the HIE-BS group.
\end{abstract}

Key words: Burst suppression; Interburst intervals; Variability; Hypoxic-ischemic encephalopathy; Status epilepticus; Pentobarbital

Burst-suppression (BS) was first reported by Swank and Watson in 1949. It has been described as a stereotyped pattern (Hughes 1986) with a quasiperiodic repetition rate (Bauer 1987). Kuroiwa and Celesia (1980) state that although the repetition rate varies between subjects, it is remarkably constant in a given patient. Recently, several authors (G.B. Young et al. 1980; R.S.K. Young et al. 1983; Rashkin et al. 1987; Lowenstein et al. 1988; Osorio and Reed 1989; Van Ness 1990) have advocated the use of pentobarbital therapy for refractory generalized tonic-clonic status epilepticus, with adequacy of therapy guided in part by the development of BS on EEG. We have observed that the duration of interburst intervals (IBIs) tended to be quite variable in these cases despite a constant rate of pentobarbital infusion impairing determination of adequate cerebral suppression, while we felt that the duration of IBIs in hypoxic brain injuries was more regular.

In this study we compare the temporal stability of IBI duration in BS induced by pentobarbital for refrac-

\footnotetext{
* Presented in part at the 1990 Annual Meeting of the American EEG Society, Houston, Texas.
}

Correspondence to: Dr. Ahmad Beydoun, University of Michigan Medical Center, Department of Neurology, EEG Laboratory, University Hospital 1B300/0036, 1500 E. Medical Center Drive, Ann Arbor, MI 48109 (U.S.A.).

Tel.: (313) 936-9030. tory convulsive status epilepticus (SE-BS) with patients in BS from hypoxic-ischemic insults (HIE-BS).

\section{Materials and methods}

\section{EEG methods}

Each EEG performed over a 3 year period from January 1st 1987 to December 31st 1989 at the University of Michigan EEG laboratory with a diagnosis of generalized BS was reviewed. Patients were excluded if less than 1 year in age, or if a clinical or electrographic seizure was recorded. The following EEG criteria were used for inclusion: bursts were defined as polymorphous complexes of high amplitude mixed activity with a duration of at least $1 \mathrm{sec}$. The IBI had to be $<10 \mu \mathrm{V}$ in amplitude, involve all channels and last for at least 1 sec. These criteria were chosen to limit the study groups to cases with definite diagnoses of BS and exclude cases with generalized periodic sharp waves or periods of relative discontinuity without complete amplitude suppression.

Ten EEGs from 10 patients with HIE-BS and 21 records from 8 patients with SE-BS met these criteria. For each EEG, the duration of the first 10 bursts and ensuing IBIs from each of 4 montages were calculated. All recordings were performed in intensive care units on 21-channel instruments using the international $10^{-}$ 20 system of electrode placement with longitudinal bipolar, transverse bipolar and referential montages. The burst and IBI durations were calculated to the 
nearest second except for records with a mean IBI duration of $<2 \mathrm{sec}$ where the duration was calculated to 1 decimal point.

\section{Statistical methods}

For each record, the mean, standard deviation, variance and coefficient of variability of the IBI durations were calculated. The coefficient of variability of IBI durations was compared between the HIE-BS and SEBS groups using the Mann-Whitney test. Simple linear regression analyses and Pearson correlation coefficients between the coefficient of variability of IBI duration and the logarithm of mean IBI duration were calculated for each group separately. Simple linear regression analyses and Pearson correlation coefficients between burst duration and ensuing IBI duration in each record and for each group were performed.

\section{Results}

The HIE-BS group consisted of 10 records from 10 patients ( 6 men, 4 women) aged 1.5-71 years (mean 38.5 years). Nine of the 10 patients died and 1 was left in a vegetative state. The SE-BS group consisted of 21 records from 8 patients ( 5 men, 3 women) aged 20-64 years (mean 39.5 years). Patients were in generalized tonic-clonic SE from various causes and were treated with phenytoin and phenobarbital before being loaded and maintained on pentobarbital. Of the 8 patients, 3 died and 5 were successfully treated and discharged.

The burst and IBI durations from a patient with HIE-BS are shown in Fig. 1 and reveal the variability of interburst durations in a single EEG. In that record, there was no statistically significant correlation $(P>$ 0.05 ) between burst duration and ensuing IBI duration. The mean, standard deviation, coefficient of variability and range of IBI durations for patients in the SE-BS

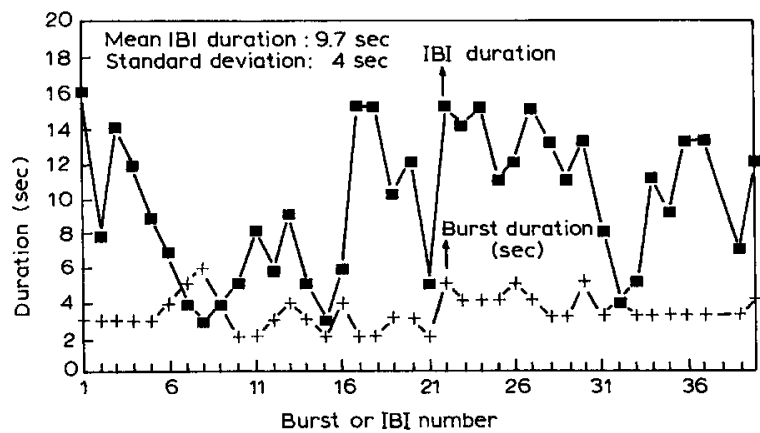

Fig. 1. Diagram of 40 bursts and succeeding interburst intervals in a patient with HIE-BS. Note the moment-to-moment variability in IBI duration and lack of correlation between burst duration and succeed ing interburst duration.

\section{TABLE I}

Mean (sec), standard deviation (sec), coefficient of variability (\%) and range of IBI durations (sec) in 21 EEGs from 8 patients in SE-BS. The mean duration represents the average duration of 40 IBIs; a-d represent sequential EEGs done in each patient.

\begin{tabular}{lrrrl}
\hline Patients & Mean & S.D. & CV & Range \\
\hline 1a & 1.079 & 0.1613 & 14.95 & $1.0-1.5$ \\
1b & 1.175 & 0.2771 & 23.58 & $1.0-1.8$ \\
1c & 3.950 & 1.6630 & 42.11 & $1-8$ \\
1d & 4.075 & 1.9530 & 47.93 & $1-9$ \\
1e & 4.325 & 2.0680 & 47.81 & $2-10$ \\
2a & 2.275 & 0.9055 & 39.80 & $1-4$ \\
2b & 3.150 & 1.5280 & 48.52 & $1-9$ \\
2c & 5.175 & 4.1440 & 80.01 & $1-19$ \\
2d & 5.367 & 2.4840 & 46.29 & $1-10$ \\
3a & 1.685 & 0.6765 & 40.15 & $1.0-3.4$ \\
3b & 3.100 & 1.2130 & 39.14 & $1-6$ \\
3c & 4.400 & 3.1360 & 71.27 & $2-13$ \\
3d & 6.950 & 5.5330 & 79.61 & $1-29$ \\
$4 \mathrm{a}$ & 1.505 & 0.4632 & 30.78 & $1.0-2.6$ \\
4b & 1.835 & 0.1791 & 9.76 & $1.3-2.2$ \\
4c & 2.850 & 1.0750 & 37.73 & $1-5$ \\
5a & 1.543 & 0.4349 & 28.19 & $1.0-2.5$ \\
$5 \mathrm{~b}$ & 3.250 & 1.5480 & 47.64 & $1-7$ \\
6 & 2.468 & 0.9092 & 36.84 & $1-5$ \\
7 & 8.525 & 5.5980 & 65.66 & $2-25$ \\
8 & 15.230 & 13.4200 & 88.13 & $2-50$ \\
\hline
\end{tabular}

and HIE-BS groups are shown in Tables I and II respectively. The mean IBI duration averaged $4.0 \mathrm{sec}$ (1.1-15.2) in the SE-BS group and $14.8 \mathrm{sec}(2.5-56.3)$ in the HIE-BS group. The lower mean IBI duration in the SE-BS group probably reflects our protocol to gradually increase the maintenance pentobarbital dosage until BS with a mean IBI duration of approximately $5 \mathrm{sec}$ is achieved.

The coefficient of variability of IBIs was not statistically different between the HIE-BS and the SE-BS groups (Mann-Whitney test, $P>0.05$ ). However, it was highly and positively correlated with the logarithm of mean IBI duration in the SE-BS group $(r=0.88, P<$

\section{TABLE II}

Mean (sec), standard deviation (sec), coefficient of variability (\%) and range of IBI durations ( $\mathrm{sec}$ ) in 10 EEGs from 10 patients in HIE-BS. The mean duration represents the average duration of 40 IBIs.

\begin{tabular}{rrrll}
\hline Patients & Mean & \multicolumn{1}{l}{ S.D. } & CV & Range \\
\hline 1 & 2.500 & 0.9871 & 39.48 & $1-4$ \\
2 & 3.575 & 0.9306 & 26.03 & $1-6$ \\
3 & 4.650 & 1.8750 & 40.32 & $2-11$ \\
4 & 6.700 & 5.4740 & 81.69 & $1-18$ \\
5 & 9.053 & 1.0380 & 11.47 & $7-11$ \\
6 & 9.675 & 3.9703 & 41.04 & $3-16$ \\
7 & 11.030 & 5.8730 & 53.27 & $1-26$ \\
8 & 18.190 & 11.9700 & 65.76 & $2-45$ \\
9 & 26.130 & 9.2380 & 35.36 & $6-41$ \\
10 & 56.280 & 34.8400 & 61.90 & $6-136$ \\
\hline
\end{tabular}




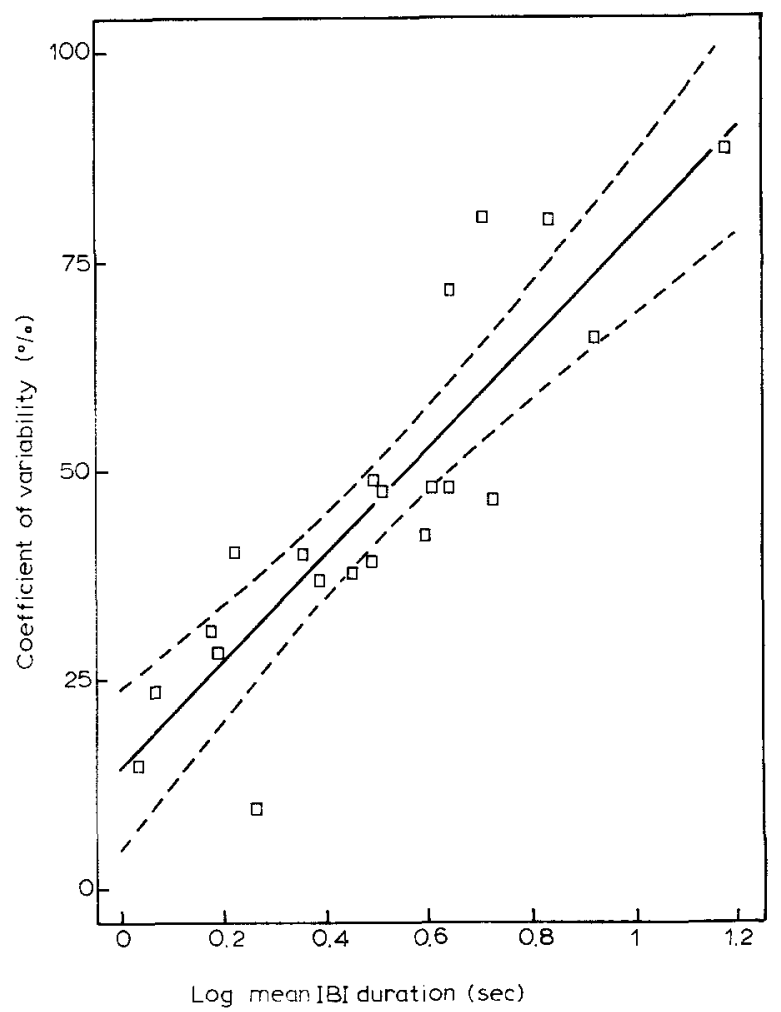

Fig. 2. Relationship between coefficient of variability of IBI duration and logarithm of mean IBI duration in 21 records with SE-BS. $\mathrm{CV}=62.99 \times \log ($ mean IBI $)+14.35(r=0.88, P<0.00001)$. Based on this function, the CV may be calculated accurately. Solid line is line of regression, dotted line $= \pm 3$ S.D.

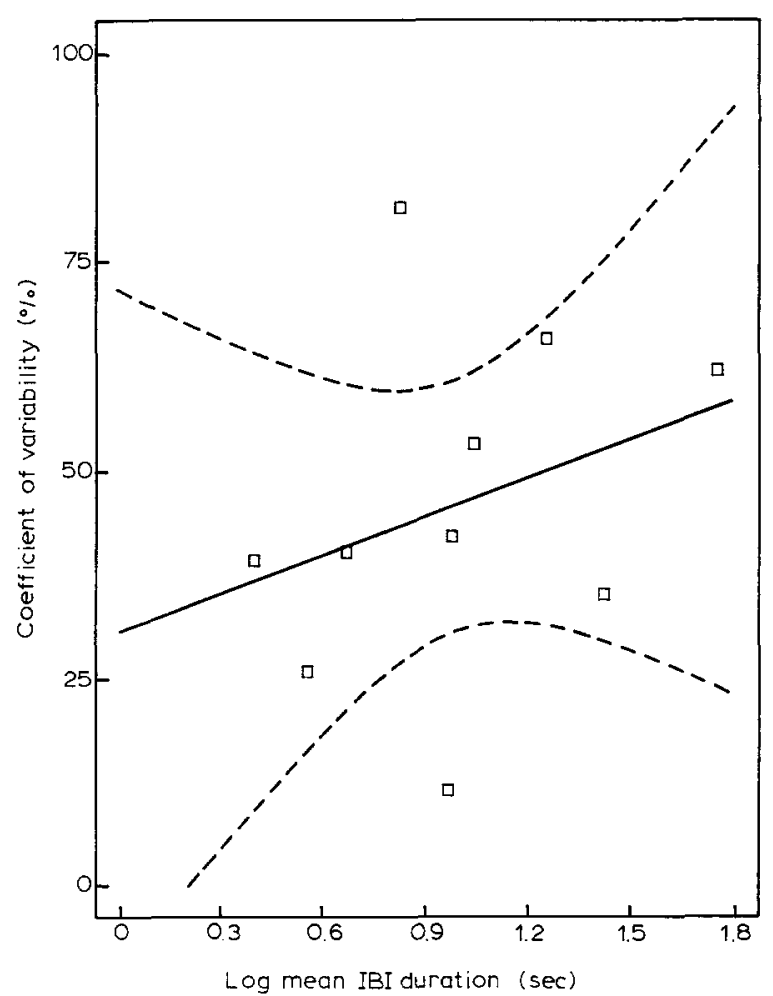

Fig. 3. Relationship between coefficient of variability of IBI duration and logarithm of mean IBI duration in 10 records with HIE-BS. Note the lack of correlation $(r=0.31, P=0.39)$.

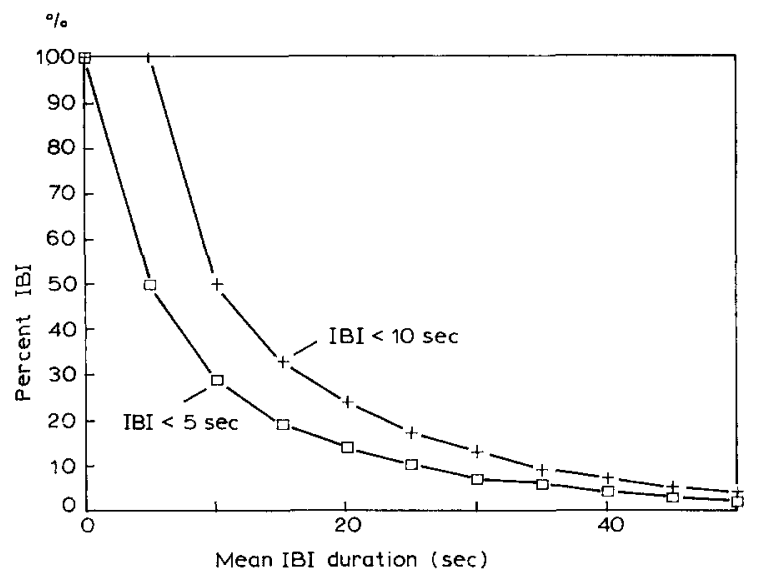

Fig. 4. Graph relating expected percentage of IBIs lasting less than 5 or $10 \mathrm{sec}$ according to the mean IBI duration in the SE-BS group.

$0.00001)$ but not in the HIE-BS group $(r=0.31, P=$ 0.39 (Figs. 2 and 3)).

There was no statistically significant correlation $(P$ $>0.05$ ) between burst duration and ensuing IBI duration in all records except for one in the SE-BS group. When mean IBI duration and mean burst duration of each record were compared in each group, there was no statistically significant correlation $(P>0.05)$ between them in either group.

We plotted the expected percentage of IBIs with a duration of less than $5 \mathrm{sec}$ and less than $10 \mathrm{sec}$ for different mean IBI durations in the SE-BS group (Fig. 4). The results were obtained according to the linear regression equation correlating the variance of IBI durations and mean IBI duration in the SE-BS group: variance $=11.04 \times($ mean $\mathrm{IBI})-30.02 \quad(r=0.98, P<$ $0.00001)$. From Fig. 4, if $10 \%$ of IBIs in a record were to last $5 \mathrm{sec}$ or less, a mean interburst duration of at least $25 \mathrm{sec}$ should be achieved. In that figure, the extrapolation of data beyond a mean IBI duration of $15.2 \mathrm{sec}$ is based upon the assumption that mean IBIs of longer duration would also fit the linear equation.

\section{Discussion}

Although the EEG pattern of BS is characteristic, there are no well established criteria for IBI amplitude or duration (Chatrian et al. 1974). Most investigators have used an IBI amplitude of $<5-10 \mu \mathrm{V}$ (Kuroiwa and Celesia 1980; Quasha et al. 1981; Grigg-Damberger et al. 1989) and a burst duration of at least $1 \mathrm{sec}$ (Kuroiwa and Celesia 1980). We found this operational definition adequate since it excludes other periodic patterns such as repetitive sharp waves or periodic discharges without suppression of background EEG.

Most investigators comment on the regularity of IBI durations in BS. However, in SE-BS great variability in 
individual IBI durations has been observed despite a constant rate of drug infusion (Kofke et al. 1989; Van Ness 1990), but without explanation. We found that the coefficient of variability of IBI duration in records from our SE-BS group was highly and positively correlated with the logarithm of mean IBI duration $(P<$ 0.00001 ). Thus, records with short IBIs tend to have an invariant repetition rate and appear to follow an endogenous rhythm. EEGs with prolonged mean IBI durations show greater variability of individual IBI durations. This seems supported by the observation of Brenner and Schaul (1990), that BS has a more regular appearance when IBIs are less than $4 \mathrm{sec}$.

Although there was no significant difference in the coefficient of variability between the SE-BS and HIEBS groups, we found no statistically significant correlation between the coefficient of variability of IBI durations and the logarithm of mean IBI duration in the HIE-BS group $(P=0.39)$. Thus records with long mean IBIs could have a relatively invariant rate or show great variability of successive IBI durations within the same record. Patient 5 in Table II illustrates the case of a relatively long mean IBI duration $(9.1 \mathrm{sec})$ with very little variability between successive IBIs within the same record (S.D. $=1.0 \mathrm{sec}$ ). Lack of variability of IBI durations in HIE-BS was reported in 2 cases by Hughes (1986) in whom the IBI durations were so regular that an artifact was initially suspected.

Since at present, there is no definite explanation for the neurophysiologic mechanisms responsible for generating a BS pattern, we can only speculate on the significance of our findings. Some investigators have proposed that BS occurs when the cortex is functionally or anatomically disconnected from the underlying white matter (Cobb and Hill 1950; Echlin et al. 1952; Henry and Scoville 1952) suggesting an "autogenicity" of deafferented cortical neurons. Barbiturates may cause a functional deafferentation of the cortex such that inherent rhythmical activity of cortical neurons will generate synchronous discharges with short IBIs. Greater cortical dysfunction will result in desynchronization of some discharges, which may then not be seen on surface EEG, as demonstrated at electrocorticography (Henry and Scoville 1952; Sperling et al. 1986). This would explain greater variability and prolongation of IBI durations. This does not explain cases of HIE-BS with prolonged mean IBI durations and little variability. Gloor et al. (1968) believe that periodicity develops following cortical and subcortical grey matter damage, that bursting is a reflection of cortical neuronal excitability to input from subcortical (Gloor et al. 1968; Lenard et al. 1976) or brain-stem regions (Fenyo and Hasznos 1964) and that the IBI is a reflection of the refractory period of cortical or subcortical/ brain-stem neurons. This hypothesis of a subcortical/ brain-stem pacemaker accounting for the BS periodic- ity could explain prolonged mean IBI durations with little variability in some cases with HIE-BS.

In view of the many disorders associated with BS, it is quite possible that more than one mechanism could lead to this pattern. The cortical and subcortical hypotheses need not be mutually exclusive. Whatever the mechanism involved, it is likely that our findings in SE-BS reflect similar and progressive affection of identical brain structures in patients with drug induced BS as shown by Eger et al. (1971). The poor correlation seen in patients with HIE-BS is probably the result of patchy and variable damage to gray and white matter in patients with hypoxic brain injury.

One other possibility to explain our findings in the SE-BS group could be related to a differential effect of pentobarbital at varying brain concentrations, with a more uniform action at low brain concentrations and greater variability at higher levels. Chronic infusion of pentobarbital in cats produced stable CSF pentobarbital levels, which varied proportionately with serum levels (Okamoto and Boisse 1975). Furthermore, it was found that the CSF pentobarbital half-life was the same as the serum half-life (Okamoto and Boisse 1975). These findings would make fluctuations in serum or brain concentration an unlikely explanation for. IBI variability. However, it cannot be excluded that the potentiation of GABA gated chloride currents by pentobarbital (Macdonald et al. 1988) could be concentration related, with a more uniform action at low brain concentrations and a more variable effect at higher brain levels.

The practical significance of our findings is that in SE-BS, great variability between successive IBIs is to be expected, especially at dosages causing a relatively prolonged mean IBI. To date, there are no published human data to determine what constitutes adequate cerebral suppression in the treatment of SE nor is it known whether a correlation exists between IBI duration and prognosis. The only data come from animal studies that have shown gradual depression of cerebral metabolism with increasing IBI durations maximal at an IBI of $30 \mathrm{sec}$ (Kassell et al. 1980). Clinically, various investigators have aimed for IBIs varying between 2 and $30 \mathrm{sec}$ (G.B. Young et al. 1980; R.S.K. Young et al. 1983; Rashkin et al. 1987; Lowenstein et al. 1988; Kofke et al. 1989; Van Ness 1990) without an adequate basis for the IBI duration chosen. Because of the variability of successive IBI durations, we suggest that future studies dealing with drug induced BS for treatment of SE report the mean IBI duration achieved in each patient and analyze mean IBI duration as one of many variables that may determine outcome.

The authors are grateful to Dr. Morton B. Brown, School of Public Health, University of Michigan for his help in the statistical 
analysis of the data, and to Miss Sally Mouilleseaux for assistance in manuscript preparation.

\section{References}

Bauer, G. Coma and brain death. In: E. Niedermeyer and F. Lopes da Silva (Eds.), Electroencephalography. Urban and Schwarzenberg, Baltimore, MD, 1987: 391-404.

Brenner, R.P. and Schaul, N. Periodic EEG patterns: classification, clinical correlation, and pathophysiology. J. Clin. Neurophysiol., 1990, 7: 249-267.

Chatrian, G.E., Bergamini, L., Dondey, M., Klass, D.W., LennoxBuchthal, M. and Petersén, I. A glossary of terms most commonly used by clinical electroencephalographers. Electroenceph. clin. Neurophysiol., 1974, 37: 538-548.

Cobb, W. and Hill, D. Electroencephalogram in subacute progressive encephalitis. Brain, 1950, 73: 392-404.

Echlin, F.A., Arnett, V. and Zoll, J. Paroxysmal high voltage discharges from isolated and partially isolated human and animal cerebral cortex. Electroenceph. clin. Neurophysiol.. 1952, 4: 147164

Eger, E.I., Stevens, W.C. and Cromwell, T.H. The electroencephalogram in man anesthetized with forane. Anesthesiology, 1971, 35: 504-508.

Fenyo, E. and Hasznos, T. Periodic EEG complexes in subacute panencephalitis: reactivity, response to drugs and respiratory relationships. Electroenceph. clin. Neurophysiol., 1964, 16: 446458 .

Gloor, P., Kalabay, O. and Giard, N. The electroencephalogram in diffuse encephalopathies: electroencephalographic correlates of grey and white matter lesions. Brain, 1968, 91: 779-802.

Grigg-Damberger, M.M., Coker, S.B., Halsey, C.L. and Anderson, C.L. Neonatal burst suppression: its developmental significance. Pediat. Neurol., 1989, 5: 84-92.

Henry, C.E. and Scoville, W.B. Suppression-burst activity from isolated cerebral cortex in man. Electroenceph. clin. Neurophysiol., 1952, 4: 1-22.

Hughes, J.R. Extreme stereotypy in the burst suppression pattern. Clin. Electroenceph:, 1986, 17: 162-168.

Kassell, N.F., Hitchon, P.W., Gerk, M.K., Sokoll, M.D. and Hill, T.R. Alterations in cerebral blood flow, oxygen metabolism, and electrical activity produced by high dose sodium thiopental. Neurosurgery, 1980, 7: 598-603.
Kofke, W.A., Young, R.S.K., Davis, P. et al. Isoflurane for refractory status epilepticus: a clinical series. Anesthesiology, 1989, 71 : $653-659$.

Kuroiwa, Y. and Celesia, G.G. Clinical significance of periodic EEG patterns. Arch. Neurol., 1980, 37: 15-20.

Lenard, H.G., Yaneza, P.L. and Reimer, M. Polygraphic recordings in subacute sclerosing panencephalitis. A study of the pathophysiology of the periodic EEG complexes. Neuropädiatrie, 1976, 7: $52-65$.

Lowenstein, D.H., Aminoff, M.J. and Simon, R.P. Barbiturate anesthesia in the treatment of status epilepticus: clinical experience with 14 patients. Neurology, 1988, 38: 395-400.

Macdonald, R.L., Twyman, R.E., Rogers, C.J. and Weddle, M.G Pentobarbital regulation of the kinetic properties of GABA receptor chloride channels. Adv. Biochem. Psychopharmacol., 1988, 45: $61-71$.

Osorio, I. and Reed, R.C. Treatment of refractory generalized tonic-clonic status epilepticus with pentobarbital anesthesia after high-dose phenytoin. Epilepsia, 1989, 30: 464-471.

Okamoto, $\mathrm{M}$ and Boisse, N.R. Effect of chronic pentobarbital treatment on blood-cerebrospinal fluid kinetics. Eur. J. Pharmacol., 1975, 33: 205-209.

Quasha, A.L., Tinker, J.H. and Sharbrough, F.W. Hypothermia plus thiopental. Prolonged electroencephalographic suppression. Anesthesiology, 1981, 55: 636-640.

Rashkin, M.C., Youngs, C. and Penovich, P. Pentobarbital treatment of refractory status epilepticus. Neurology, 1987, 37: 500-503.

Sperling, M.R., Brown, W.J. and Crandall, P.H. Focal burst-suppression induced by thiopental. Electroenceph. clin. Neurophysiol, 1986, 63: 203-208.

Swank, R.L. and Watson, C.W. Effects of barbiturates and ether on spontaneous electrical activity of dog brain. J. Neurophysiol., 1949, 12: 137-160.

Van Ness, P.C. Pentobarbital and EEG burst suppression in treatment of status epilepticus refractory to benzodiazepines and phenytoin. Epilepsia, 1990, 31: 61-67.

Young, G.B., Blume, W.T., Bolton, C.F. and Warren, K.G. Anesthetic barbiturates in refractory status epilepticus. Can. J. Neurol. Sci., 1980, 7: 291-292.

Young, R.S.K., Ropper, A.H., Hawkes, D., Woods, M. and Yohn, P. Pentobarbital in refractory status epilepticus. Pediat. Pharmacol., 1983, 3: 63-67. 